\title{
Supersonic Engine Inlet Tone Noise Radiation
}

\author{
David B. Stephens ${ }^{1}$ and Christopher J. Miller ${ }^{1}$ \\ NASA Glenn Research Center, Cleveland, OH, 44135 \\ Paul E. Slaboch ${ }^{1}$; Jonathan Brown \\ University of Hartford, West Hartford, CT, 06117 \\ Jonathan M. Celestina \\ University of Detroit Mercy, Detroit, Michigan, 48221
}

A computational and experimental acoustic analysis was conducted on a supersonic engine inlet geometry with a spike center body and an auxiliary inlet. Simulations performed using two different commercial acoustics software packages were compared to a scale model experiment conducted using an ultrasonic fan noise simulator. Both the experiment and simulations were run at discrete circumferential mode and frequency combinations to identify strengths and weaknesses of each method. For cases where a single azimuthal mode was well cut on throughout the length of the duct, reasonable agreement was found between the three methods. In cases with more complicated acoustic effects, the experimental results became intractable when considered alone and the simulations were needed for interpreting the results. The peak sound level and the peak angle of the far field radiation vary widely when changing modes and frequencies. The objective of the study is to develop a validated workflow for simulation of fan tone noise through supersonic inlet geometries for community noise predictions.

\section{Introduction}

Commercial supersonic aircraft are of current interest as NASA pursues a Low-Boom Flight Demonstrator (X-59A) and several companies are pursuing supersonic business jets. It is assumed that any new aircraft would be certified using existing noise regulations that apply to subsonic aircraft. While the propulsion system is designed for peak efficiency during cruise at supersonic Mach numbers, it must also operate at subsonic speeds during take-off and landing operations around airports. The jet exhaust flow is frequently the most dominant engine noise contribution, but fan noise is an important source as slower cruise speeds are considered and the jet noise becomes quieter.

The NASA Commercial Supersonics Technology (CST) project sponsored a study by GE Aviation, Lockheed Martin and Stanford University [1] that developed a concept vehicle called the LM1044, a Mach 1.7, 80-passenger tri-jet. Their study indicated that fan noise was a contributor to vehicle noise at some conditions, and specifically that the auxiliary inlet was a particular concern. The auxiliary inlet is an additional opening that is needed to provide additional mass flow to the engine when operating at low flight speeds. This process introduces flow distortion into the fan, which adds another noise generating mechanism. The auxiliary inlet also provides a noise leakage path for fan noise to radiate to an observer on the ground. Finally, the supersonic spike inlet requires radial struts to hold up the center body, which are another source of flow distortion. A render of the LM1044 showing the engine inlets in cruise configuration is given in Figure 1.

\footnotetext{
${ }^{1}$ AIAA Member
} 


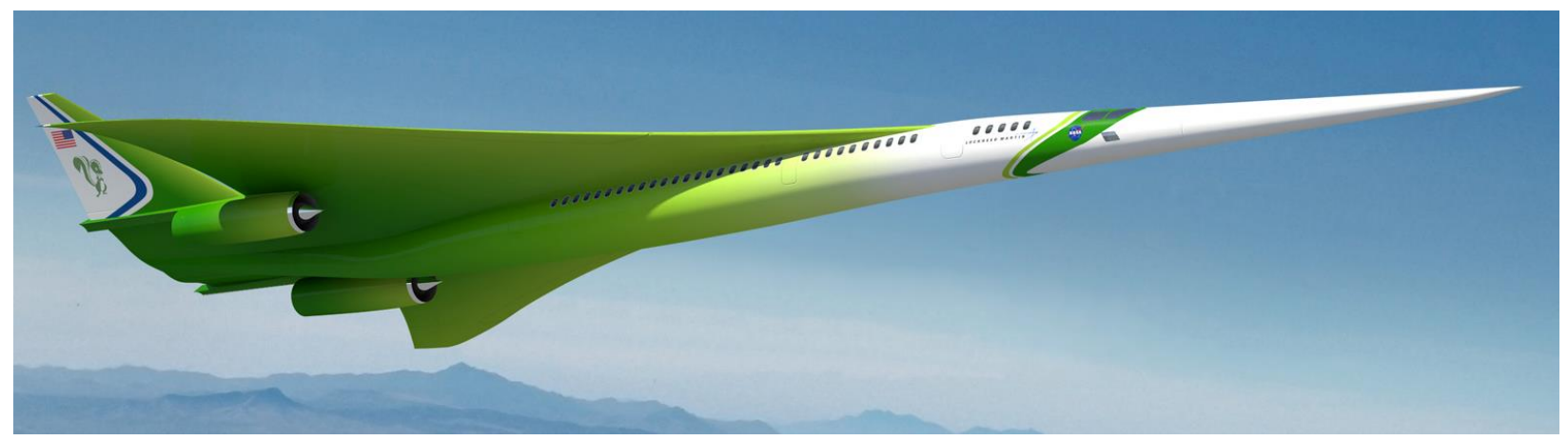

Figure 1 - NASA/Lockheed Martin LM1044 showing spike engine inlets in cruise configuration.

A study led by NASA Glenn Research Center [2] considered the Low-Boom Flight Demonstrator concept geometry. Compared to the LM1044, this aircraft is a single occupant, single-engine design with a Mach 1.6 flight speed. The report coupled the aircraft geometry and CFD to an engine model representing a General Electric F404 turbofan. The inlet used was an axisymmetric spike with an idealized axisymmetric auxiliary door, similar to that from the LM1044 study. In this design, the upstream part of the inlet slides forward to create an additional opening for air to enter the engine. Among other findings, it was determined that the appropriate flight speed for switching between open and closed auxiliary inlet configurations was Mach 0.6. Therefore, the open auxiliary inlet configuration is the appropriate inlet geometry for community noise assessment.

The present report describes the geometry being considered and the motivation for conducting an experiment without airflow. An experiment using a fan noise simulator was conducted in the Acoustical Testing Laboratory (ATL) at NASA Glenn Research Center (GRC). The experimental setup is briefly described and measurement results are given. A workflow for Actran is discussed, where the methods used to import the inlet geometry from 3-D CAD software and conduct 2-D azimuthal simulations is described. Details of complementary 3-D acoustic simulations conducted in COMSOL Multiphysics ${ }^{\circledR}$ are also given. Results from the three methods are presented together for examination. Finally, a discussion of the methods and results is presented.

\section{Geometry}

The axisymmetric spike inlet geometry was supplied by the authors of [2] for use in the present study. This geometry features an auxiliary inlet but no struts supporting the center body. The geometry was scaled such that the downstream end of the inlet had an annular geometry with an outer flow path diameter of $149 \mathrm{~mm}$ (5.87 inches) and an inner flow path diameter of $44.7 \mathrm{~mm}$ (1.76 inches). This is the location of the aerodynamic interface plane, where the inlet connects to the engine. This physical size was chosen to match the annular outer diameter of an existing ultrasonic fan noise simulator, which will be described in Section 3. This gives a scale factor of 11 for the LM1044 vehicle with its $1650 \mathrm{~mm}$ (65 inch) fan diameter and a scale factor of 6 for the $890 \mathrm{~mm}$ (35 inch) F404 engine fan used in [2]. Frequencies of interest will scale linearly, so to consider a tone of $2500 \mathrm{~Hz}$ full scale, model scale simulations would be at frequencies of $15 \mathrm{kHz}$ to match the scale factor of 6 or $27.5 \mathrm{kHz}$ for the scale factor of 11 .

Fittings were added to the CAD model for attaching to the existing parts. The scaled inlet was adapted for 3-D printing on a stereo lithography machine, and was produced in three parts. Thin steel pins, outer diameter $3 \mathrm{~mm}$ ( 0.125 in) were used to hold the center body in place and connect to both the upstream and downstream portions of the inlet. The model was only designed for acoustic testing in a quiescent anechoic chamber, so there are no air loads. The geometry is shown in Figure 2. 


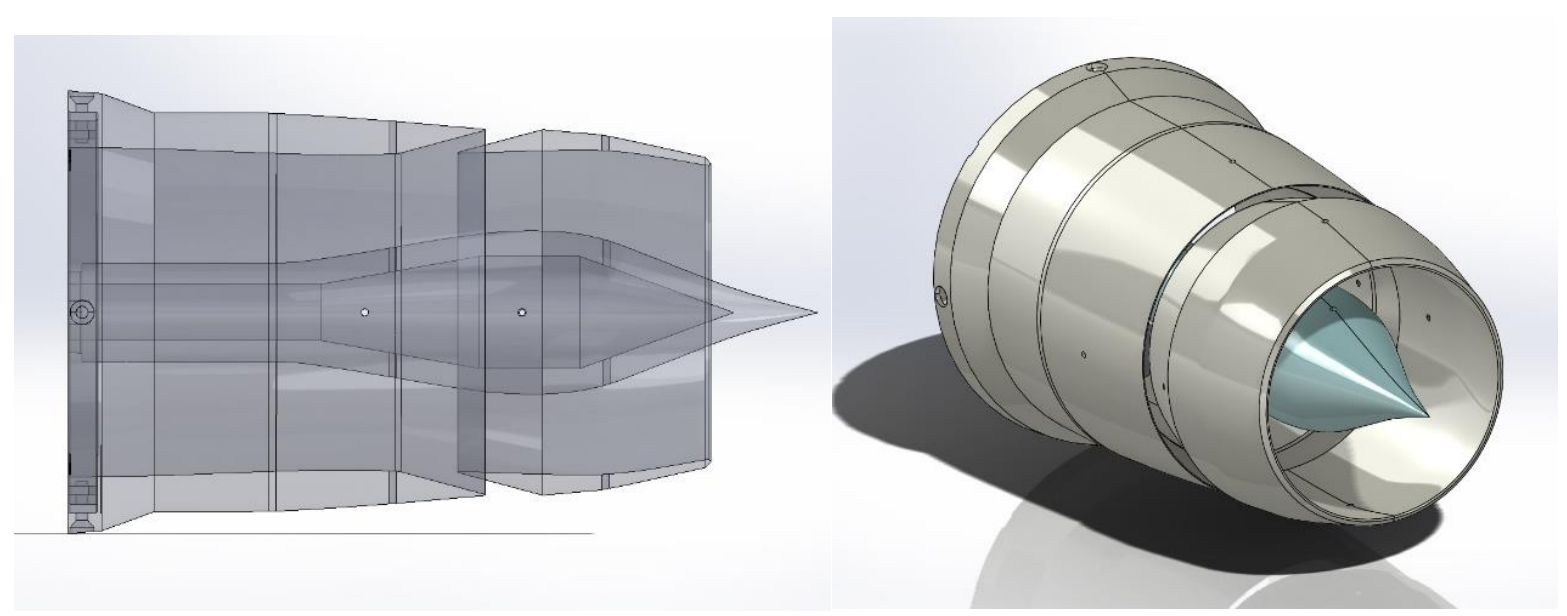

Figure 2 - Axisymmetric spike inlet geometry with auxiliary inlet used in the present study.

This idealized or axisymmetric study provides a starting point for building up complexity and realism. The axisymmetric design also simplifies the interpretation of experimental results. Finally, the axisymmetric design can be reduced to a 2-D problem for simulation, greatly reducing computational costs. A more realistic inlet would require support struts for both the forward portion of the inlet and the center body, plus actuation mechanisms. All of these could have a significant effect on the noise radiation as they would break the symmetry of the annular duct.

\section{Experimental Setup}

The Ultrasonic Configurable Fan Noise Simulator (UCFANS) was designed and built as part of the Environmentally Responsible Aviation (ERA) project [3]. As used for ERA, the device incorporated 36 ultrasonic acoustic drivers that generate rotating acoustic modes inside the duct, similar to those generated by an aircraft engine fan. The requirement to host 18 of the drivers from the inner part of the annulus meant a very large hub to tip ratio, which would have been incompatible with the converging/diverging shape of the spike inlet. The inner drivers were removed and only the outer 18 were used for the present test. The drivers are placed at an angle to allow a greater density at a given axial location. The positive spinning mode rotation direction is defined as clockwise, forward looking aft. This is the direction that the face of the drivers points. The device was previously used to study noise shielding due to airframe or wing configurations [4] [5], and the use for supersonic inlet acoustics is a new application. Fan noise simulators can be a very useful alternative to rotating machinery for detailed noise studies [6]. Compared to computational methods, experiments may lend themselves to larger domain sizes or higher frequencies that may take a large amount of finite element grid nodes, such as shielding studies. Many realistic geometries do not lend themselves to 2-D solutions, such as square inlets or non-axisymmetric auxiliary inlet designs.

Upstream of the noise generating section, a series of 72 microphones measures the noise in the duct. These were Panasonic Omnidirectional Back Electret Condenser Microphone Cartridges, WM-64C/64K series. A spacer was added between the acoustic drivers and the microphone array, so that a constant cross-section diameter is maintained before and over the mode measurement ring. It was hoped that this would allow evanescent acoustic modes to decay, rather than being measured by the microphones. After passing through the mode measurement ring, the noise then propagates out of the inlet. The schematic of the experimental rig is shown in Figure 3. 


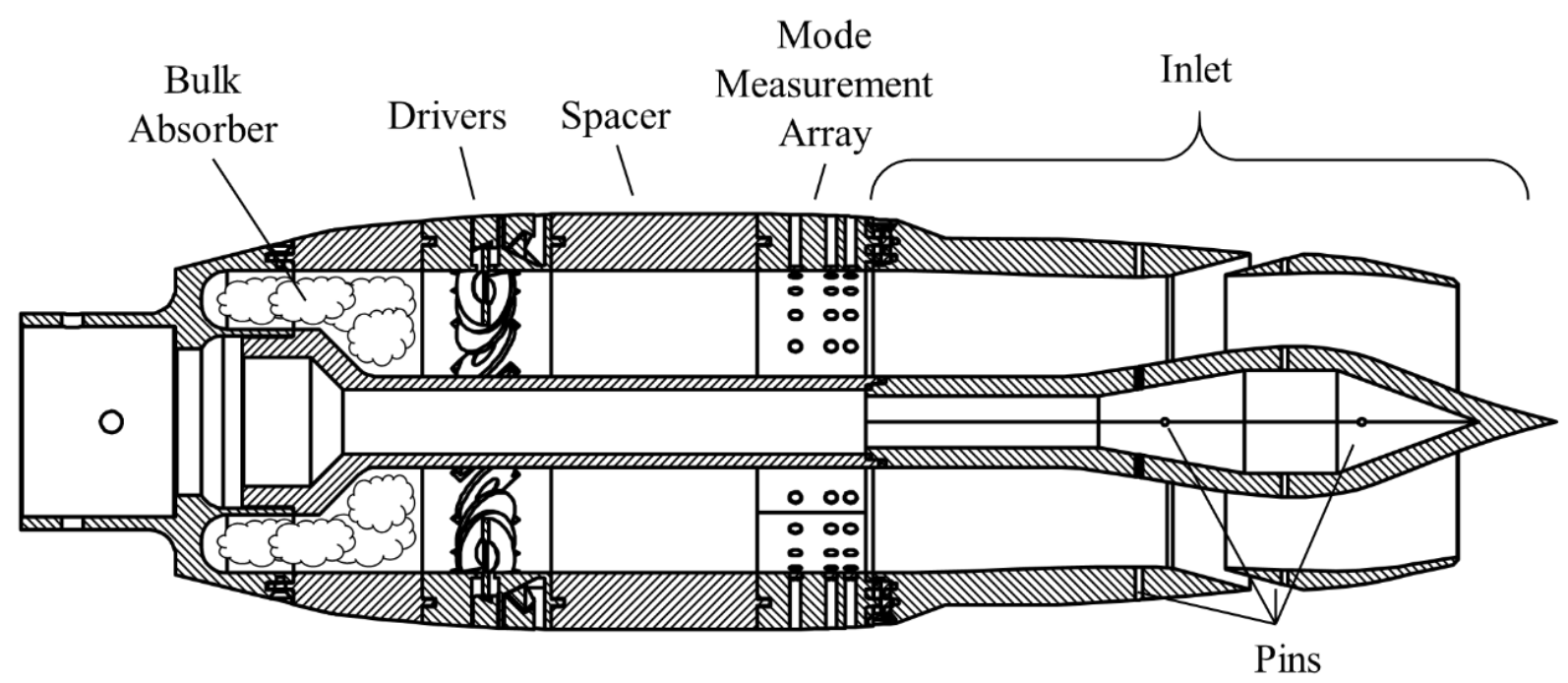

Figure 3 - UCFANS adapted for supersonic inlet propagation assessment. Section of 18 ultrasonic drivers is shown as (9), 72 measurement microphones shown as (5).

The electronics that operated the fan noise simulator were largely similar to those used during the ERA testing. The analog output electronics was a National Instruments PXI 1044 chassis with PXI 6733 cards, three of which were synchronized and operated at a sample rate of $100 \mathrm{kHz}$. A 19 -channel, $100 \mathrm{kHz}$ periodic WAV file was generated using Matlab, and this was read by LabVIEW and written to the analog output channels. The sample was one second long, which was small enough to fit in the buffer of the analog output cards and thus could be repeated indefinitely when mode generation was desired. The first 18 signals were sent to a set of ED1 Electrostatic Speaker Drivers by Tucker-Davis Technologies, which drove the ultrasonic speakers. The $19^{\text {th }}$ output channel was used as a phase reference and recorded on the other data systems used in the experiment.

A set of nine custom 8-channel microphone amplifiers was provided by Channel Island Acoustics. These were used for the 72-microphone mode measurement array on the outer duct wall. A National Instruments PXIe-1082 chassis with PXIe-4498 analog input cards was used to record the 72 microphone channels of the mode measurement array, along with the phase reference signal. The drivers and microphones of the mode measurement system were individually calibrated on a bench top before assembly into the model.

The assembled fan noise simulator was operated in the Acoustical Testing Laboratory at NASA GRC [7] [8], which is a configurable anechoic chamber with inner dimensions of $5.2 \mathrm{~m}$ by $5.2 \mathrm{~m}$ by $6.4 \mathrm{~m}$ (17- by 17- by 21 - feet). The installation is shown in Figure 4, where the model is pictured with the traversing microphone array overhead. During testing, the model was wrapped in sound absorbing material to reduce the chance of noise leakage through gaps in the assembly. 


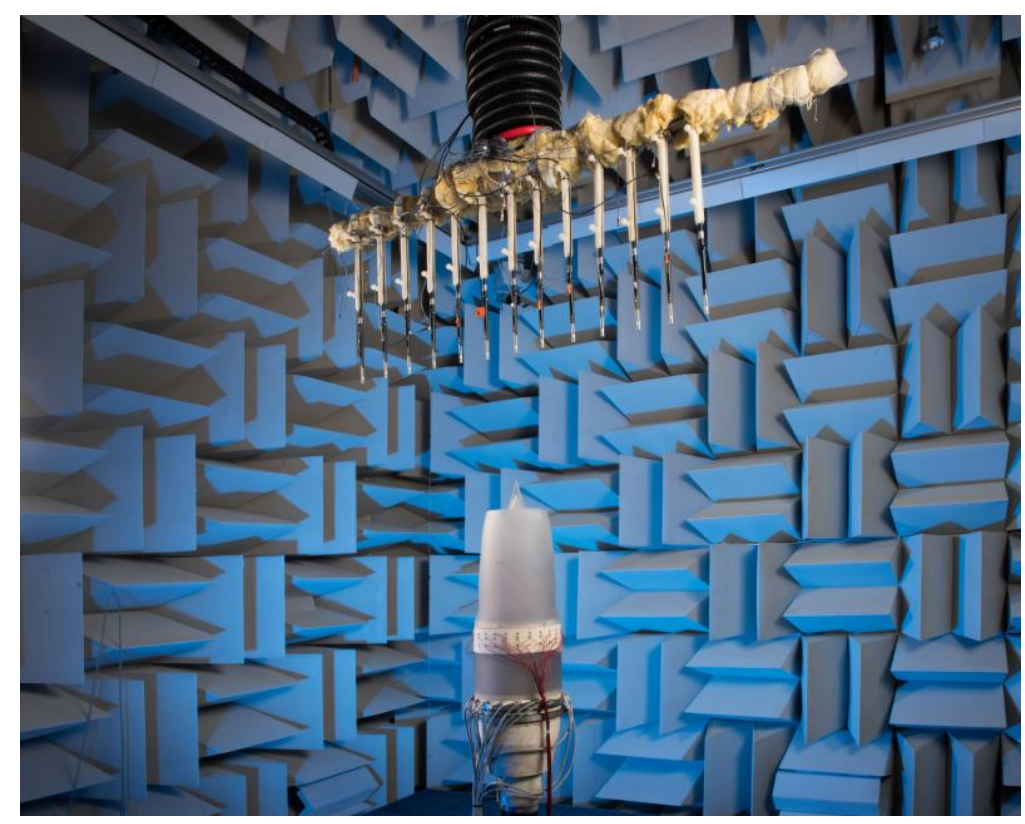

Figure 4 - Fan noise simulator with supersonic inlet installed in the Acoustical Testing Laboratory. Overhead traverse with 13 microphones is also pictured. Note that inlet shown is the axisymmetric spike inlet with the auxiliary inlet closed. The overhead microphone array is also lower than was used for the data presented here.

The traversing microphones were PCB Type 378C01 units and were recorded using an HBM Gen2i Genesis. The radiated sound was surveyed using the ATL overhead traverse rake. Microphones were spaced $76 \mathrm{~mm}$ (3 inches) apart in the y-direction on the microphone holder. Motion was commanded in the $\mathrm{x} / \mathrm{y}$ plane and sweeps were taken in the $\mathrm{x}$ direction with stops every $76 \mathrm{~mm}$ (3 inches). The resulting measurement surveys were at $x=+/-2130 \mathrm{~mm}$ (84 inches), for $y=+2130 \mathrm{~mm}$ ( 84 inches) and $-2057 \mathrm{~mm}$ (81 inches), at a $76 \mathrm{~mm}$ ( 3 inches) grid spacing in all directions. The center of the model was along the $\mathrm{z}$ axis $(x=y=0)$ and the height above the spike inlet exit lip was $1194 \mathrm{~mm}$ (47 inches). This height was with the microphone array nearly retracted to the ceiling, to give the best representation of the acoustic far field. The resulting survey grid was 57 points in the $\mathrm{x}$-direction and 56 points in the y-direction for 3192 total grid points. Two seconds of data was recorded for all traversing microphones while the array was stopped. The complete four-sweep survey took approximately thirty minutes.

Because the microphones would not necessarily be pointed normal to the sound propagation direction during a traverse, a frequency-dependent amplitude correction was developed. Two different microphones were placed on a turntable and rotated through a full rotation in 10-degree increments while a tweeter emitted a white noise signal. The measured sound spectrum at all angles was compared to the one measured when the microphone was pointed directly at the speaker, and a frequency-dependent correction was determined. The correction was found to be a relatively smooth function of angle and frequency. Corrections were applied to the measured data using a look-up table, but a polynomial fit could be used with only slightly reduced accuracy. The microphone directivity correction is shown in Figure 5. 


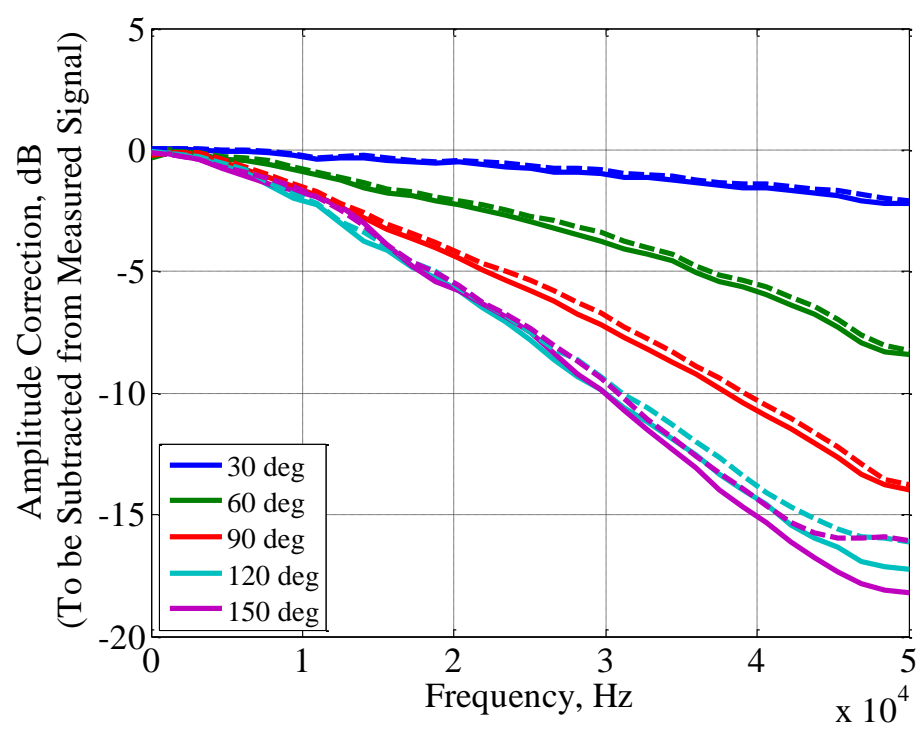

Figure 5 - Microphone directivity correction as a function of frequency. S/N 108598 shown solid and S/N 108599 shown dashed.

The time series recording for each microphone channel was processed using Matlab to give narrowband pressure spectral density (PSD). The level of the tone of interest was computed by integrating the area under the PSD curve at a few bins around the frequency of interest. This tone level was corrected for microphone sensitivity, microphone directivity, and atmospheric absorption.

A polar angle directivity was extracted from the overhead survey by converting the 2-D Cartesian survey coordinates to spherical coordinates. The amplitudes were adjusted for spherical spreading to give a "1foot lossless" amplitude, mapping the measured tone amplitude from the rectangular survey plane to the surface of a sphere. A single directivity curve was approximated from the resulting point cloud by using a moving average filter, where the average is taken in linear pressure before being converted back to decibels. Results from the experimental survey will be shown in Section 9.

\section{Actran Computational Methods and Setup}

The axisymmetric inlet geometry described in Section 2 was adapted for a 2-D axi-periodic solution in Actran TM, a finite element acoustic tool developed by Free Field Technologies. The spike inlet is an axisymmetric geometry that lends itself to using the built-in 2-D axisymmetric analysis, where the axisymmetry order can be specified to restrict the solution to a particular azimuthal mode. This simplifies the solution to a much smaller computation and therefore requires fewer resources than a 3-D simulation.

The 3-D CAD models were imported into SolidWorks and a two dimensional profile was acquired of each geometry. This profile was augmented with the acoustic field that will be computed in Actran, which is a semicircular region of radius $300 \mathrm{~mm}$ (11.8 inches), approximately 4 duct diameters, centered on the middle of the spike. The 2-D SolidWorks model is shown in Figure 6. This geometry was then exported into ANSYS, where a mesh for flow simulations was generated along with associated 1-D boundary meshes for the solid surfaces, the fan face and the radiation boundary. These meshes were identified and named in ANSYS and then exported for use with Actran. Once the geometry was in Actran, the element size of the mesh was then refined to target a maximum mesh size of $1 \mathrm{~mm}$, which corresponds to 32 points/wavelength at $10 \mathrm{kHz}$. This is overly dense for the simulations presented in the present report, but is needed for simulating frequencies of interest for full sized geometries. The mesh that was created for 
the auxiliary inlet geometry discussed in this report had a node mean edge length of $0.57 \mathrm{~mm}$ and 95980 nodes. The computational domain for the pressure field is designed to meet the recommended field map size of 2-3 wavelengths from the body to ensure accurate far field computations.

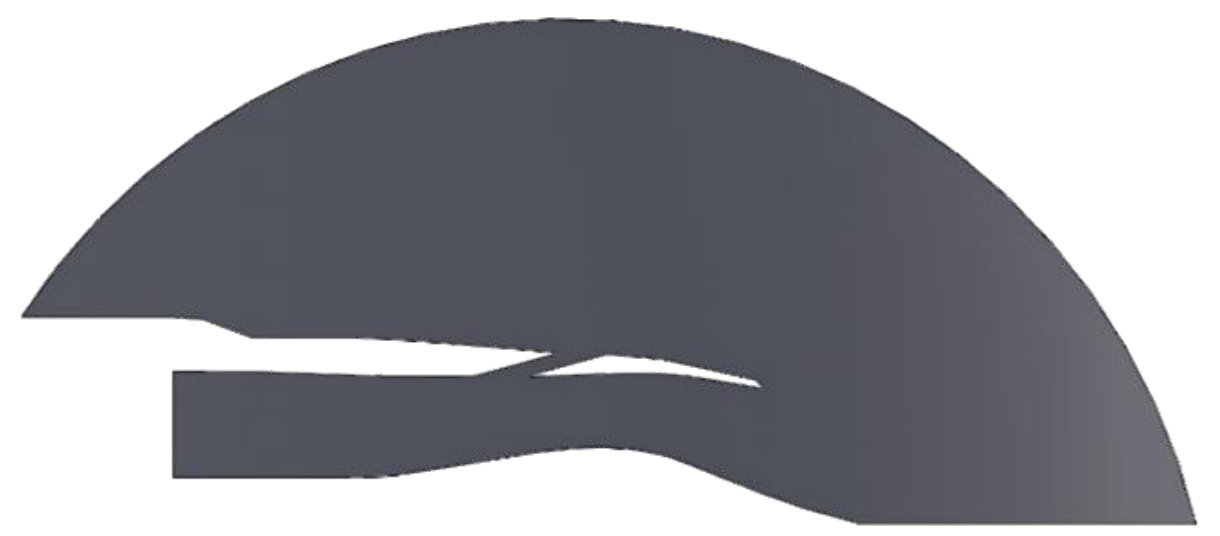

Figure 6 - SolidWorks 2018 computational 2-D geometry with the auxiliary door opened.

The directivity patterns of the radiated acoustics were determined in the far field using 121 microphones, which were set up at a $1.0 \mathrm{~m}$ radius from the center of the fan plane and from the upstream axis to $120^{\circ}$ downstream. Note that this is a different center and radius than the finite element domain the pressure field is solved on and the simulated microphone locations are not pictured in Figure 6 . The radiated acoustics were calculated from the edge of the basic domain to the far field via the infinite elements method to compute the acoustic propagation.

\section{COMSOL Numerical Simulation}

A complementary simulation was conducted using the commercial software package, COMSOL Multiphysics ${ }^{8}$ [9]. COMSOL was used to perform an acoustic pressure (acpr) analyses in which the ultrasonic drivers used in the experiment were directly modeled in 3-D, along with such details as the pins holding the model together. The CAD geometry, as seen in Figure 3, comprising the inlet, center body, microphone-array instrumented duct, driver section with individual drivers, and base, is used to create a three-dimensional COMSOL model of the inlet assembly, after some de-featuring. All body surfaces are modeled as hard-wall. In the model the driver faces have an applied pressure: +1 Pa towards the inlet, and $-1 \mathrm{~Pa}$ away from the inlet. The amplitude on each driver is multiplied by the complex phase at the driver center, e.g. $+1 \mathrm{~Pa} *(\cos (m \theta)+j \sin (m \theta))$, taking into account the circumferential mode order $m$ that is being simulated. Simulated modes spin clockwise forward looking aft. The drivers are assumed to respond ideally, with calibrations for frequency-dependent driver amplitude response handled in the experiment.

In the duct opposite to the inlet, behind the drivers, the model used a perfectly matched layer where the experiment had the duct stuffed with bulk absorber. The spherical region outside the inlet has a radius of $300 \mathrm{~mm}$ (11.8 inch) centered at the main inlet plane when the auxiliary slot is closed. This domain extent matches the grid used in Actran, although a separate sizing study was performed in COMSOL. The COMSOL model and grid is seen in Figure 7. A spherical wave radiation condition is applied to the outer boundary of the finite element method (FEM) domain, and an exterior-field calculation is used to propagate the pressures on the spherical boundary to the far field locations. In the FEM domain the model used the default quadratic elements on a free tetrahedral mesh with a maximum element spacing set to 
four per wavelength for each frequency. The radiated sound was calculated on at a far field arc from the axis back to $120^{\circ}$, as well as at planes designed to mimic the traverse planes used in the experiment.

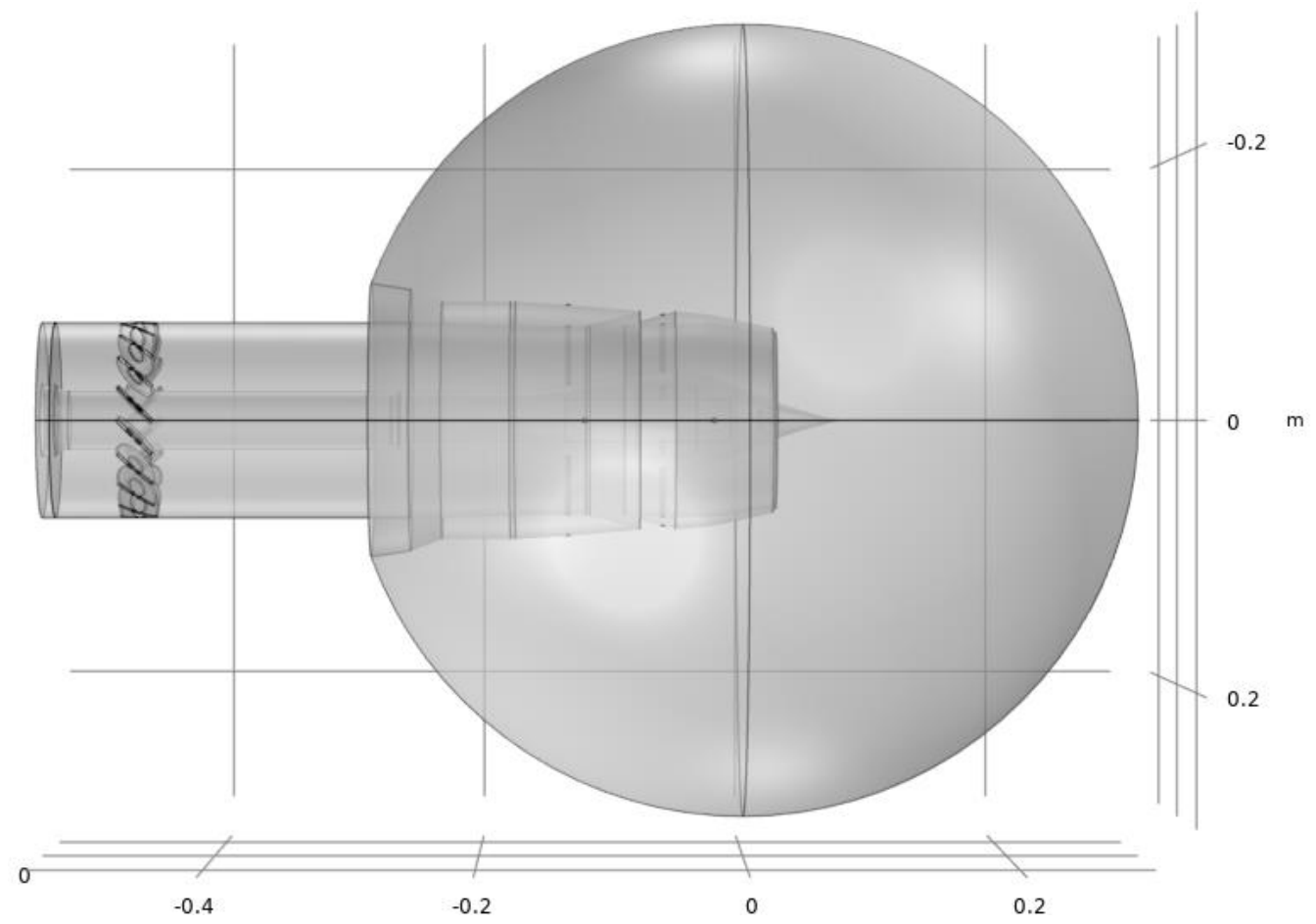

Figure 7 - COMSOL grid showing annular duct, spike inlet, drivers, auxiliary inlet and spherical solution grid region.

\section{Mode and Frequency Cases Considered}

The present work describes a set of validation cases performed using specific mode and frequency combinations that were chosen to overlap the capabilities of Actran, the experiment, and a 3-D simulation performed using COMSOL. Initial cases tested with the experiment revealed a surprising difficulty in measuring a comprehensible radiation pattern, even when only one mode-frequency combination was targeted for generation. To improve confidence in the experiment, a set of specific modes and frequencies was chosen for study. The goal was to limit the number of cut-on modes and frequencies and generate a simpler radiated sound field. The purpose of the experiment was to simulate spinning modes in the model, so the $m=0$ case was determined to not be of interest. A lower frequency set of measurements was made at $m=2$, with frequencies of 2500 to $5000 \mathrm{~Hz}$, in $500 \mathrm{~Hz}$ increments. A higher frequency set was with $m$ $=6$, and frequencies of 6500 to $10,000 \mathrm{~Hz}$, in $500 \mathrm{~Hz}$ increments. The low end of these frequencies was chosen such that the specific azimuthal mode was barely cut on. The high end was chosen where the second radial mode cut on.

In order to get an idea for how the duct modes might change as they propagate down the varying geometry of the axial spike inlet, the cut-on frequency was computed for both of the azimuthal modes tested, $m=2$ and $m=6$. This calculation was done for two radial modes, $r=0$ and $r=1$. The cut-on frequency is a function of both inner and outer duct diameters, and so the propagation angle for a certain mode can be expected to change as the duct cross-section changes. For $m=2$, the first radial mode $(2,1)$ is calculated to cut on at $4563 \mathrm{~Hz}$, so only the case at $5000 \mathrm{~Hz}$ includes the possibility of two radial 
modes. For $m=6$, at the inlet exit (left hand side of Figure 8), the first radial mode (6,1) cuts on at 8526 Hz. At the auxiliary inlet, however, the sudden expansion of the outer radius causes the cut-on frequency to drop by several hundred Hertz. For a tone at $8500 \mathrm{~Hz}$ and $m=6$, for example, scattering into the first radial mode is a distinct possibility.

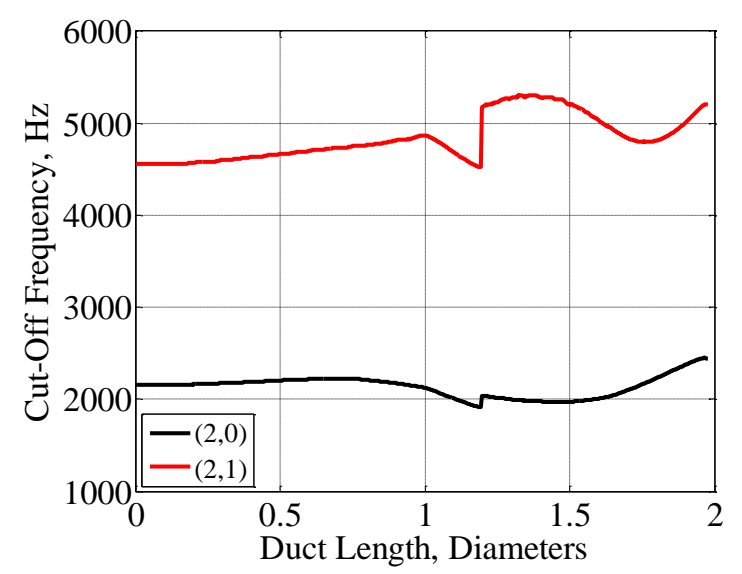

a) Mode (2,0) and (2,1) cut-on frequencies vs duct length.

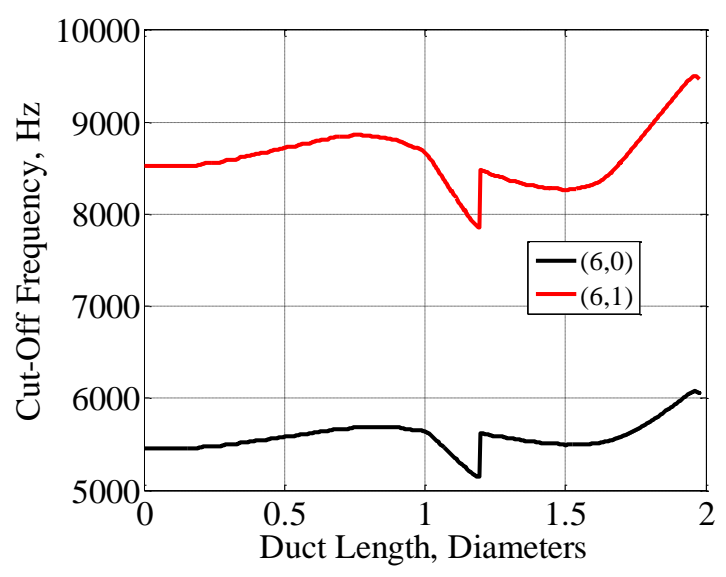

b) Mode $(6,0)$ and $(6,1)$ cut-on frequencies vs duct length.

Figure 8 -Cut-on frequencies of annular duct modes as a function of inlet geometry. The opening of the inlet is at approximately 2 diameters and the fan interface plane is at 0 diameters.

\section{Compiled Results}

The comparison of multiple diagnostic methods provides additional insight into the various capabilities, limitations and built-in assumptions incorporated. Of the 14 mode/frequency combinations tested for the present report, only three are shown for brevity. They are presented as three sets of six charts, from Figure 9, a) through f) to Figure 11, a) through f). Subfigures a) and b) show the pressure field generated by Actran and COMSOL respectively. This is useful for understanding the relationship between the in-duct sound levels and the radiated sound. Subfigures c) and d) show the overhead sound levels measured in the experiment, 3" spacing, and computed by COMSOL, 0.5 " spacing, respectively. Subfigure e) shows the in-duct mode identification ring results and subfigure f) shows the radiated sound directivity from all three methods.

The first result shown is for $m=2$ and $2500 \mathrm{~Hz}$. For this case, it was determined that large amplitude standing waves exist in the duct. It is partially a coincidence that this is evident in both simulations, as the length of duct between the sound source and the duct exit is different between Actran and COMSOL. It seems the barely cut-on spinning mode strongly reflects off of the main inlet. The wave pattern in the duct shows node lines at constant duct axial location, clearly seen in the 3-D COMSOL solution. The sound field radiated to the overhead measurement array is not the expected circular result, but instead shows several additional nulls and the overall radiation pattern does not show a strong azimuthal lobe. These imperfections would be more difficult to interpret if a smaller survey had been used. An axisymmetric model might be expected to generate perfectly axisymmetric measurements, but the steady tone leads to perfect constructive and destructive interference which can cause significant irregularities in the measured tone level. The mode measurement ring identifies a very strong $m=2$ mode pattern. The close match in amplitude for each ring suggests that the mode is generated cleanly, but the COMSOL result indicates a 
standing wave pattern that is parked over the measurement ring. The radiated sound directivity comparison between the simulations and the experiment is poor.

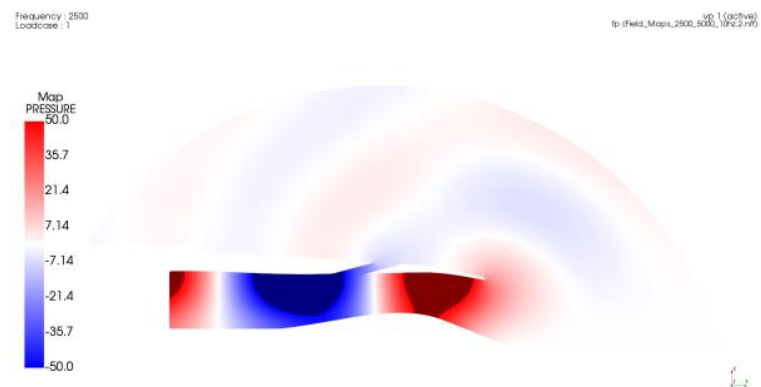

a) Actran pressure field

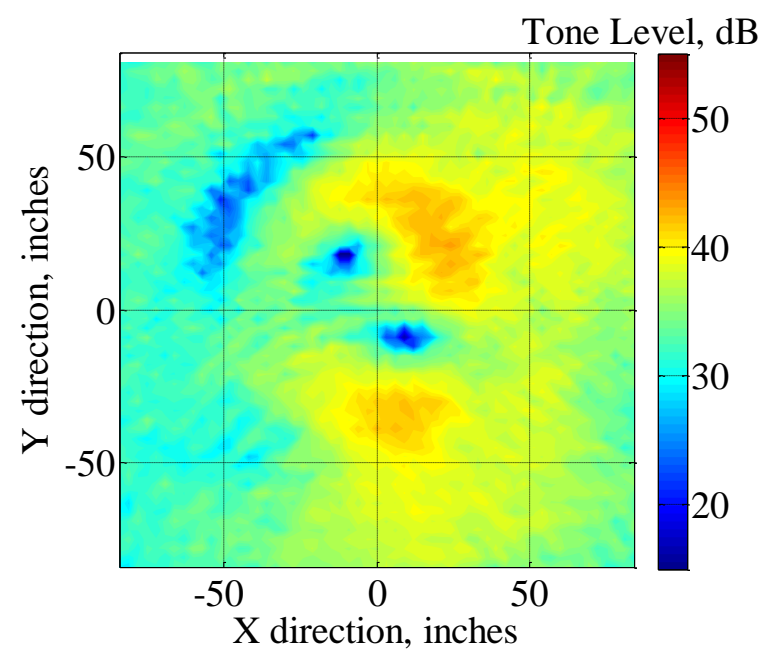

c) Experiment overhead survey

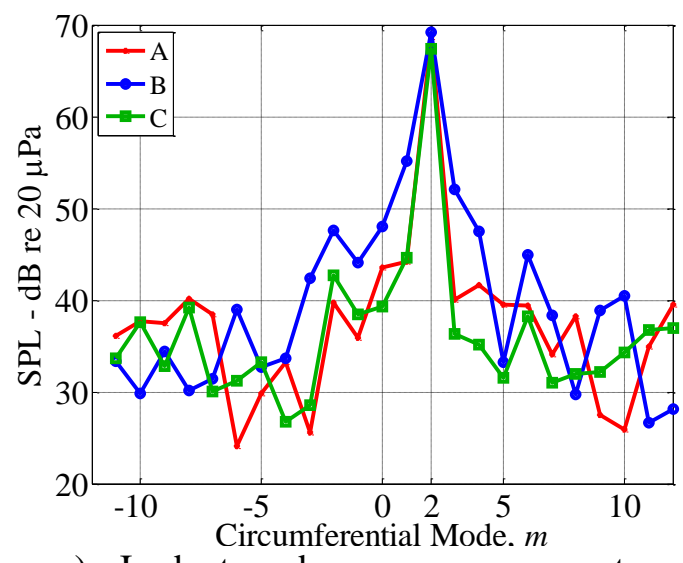

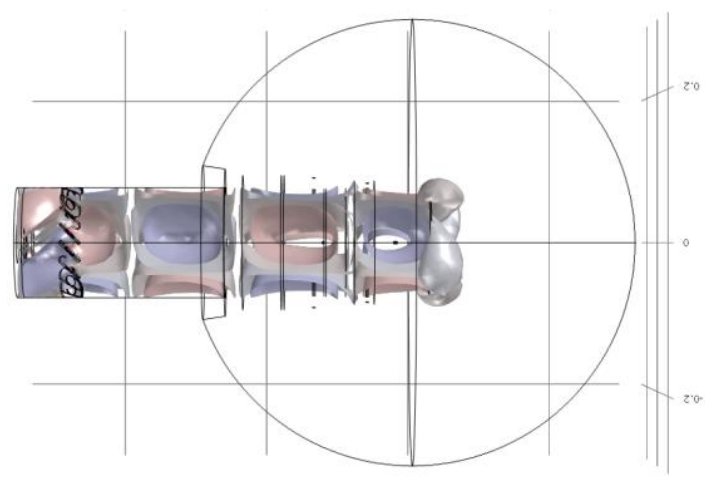

b) COMSOL pressure field

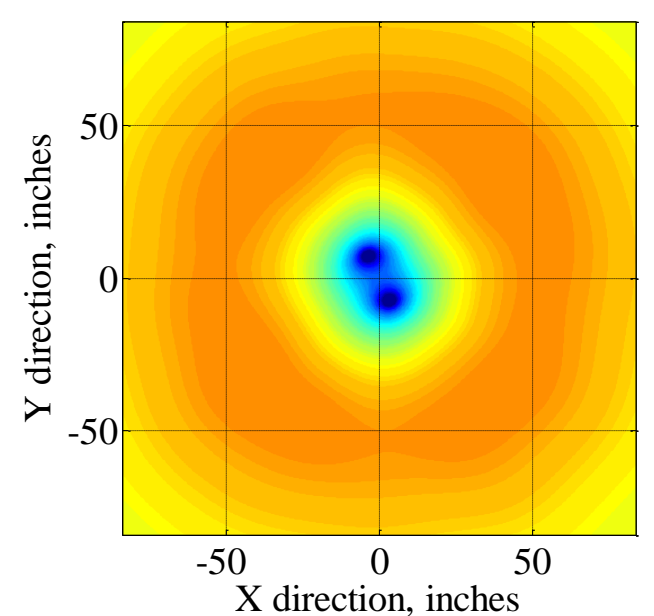

d) COMSOL overhead survey

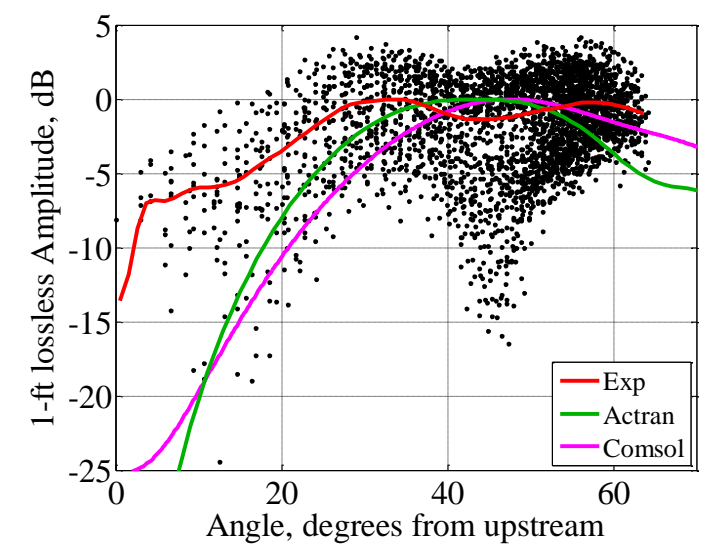

f) Radiated sound directivity

Figure 9-Combined diagnostic results for $m=2,2500 \mathrm{~Hz}$. 
A similar set of results is compared for $m=2$ and $3500 \mathrm{~Hz}$, given in Figure 10. The Actran pressure field, seen in subfigure a) shows a null along the axis of the duct, along with one strong lobe pattern radiated forward. The in-duct pressure does not generate strong standing waves and the sound pattern in the duct is much more consistant with the expected spiral shape, seen in subfigure b). The overhead sound field measured by the experiment also shows the null in the radiated sound along the axis of the duct, plus a circular radiation pattern. There is noticable scatter in the measurements, but nothing that stands out as an additional feature in the directivity. The null in the center is not as pronounced as in COMSOL, but this may be due to imperfections in the experimental geometry, or due to reflections from the measurement array. The background noise for the facility at $3500 \mathrm{~Hz}$ is well below the measured $15 \mathrm{~dB}$, so this is not a signal-to-noise ratio problem. As seen in Figure 10 e), the in-duct mode measurement is clean, with $m=$ 2 having $15 \mathrm{~dB}$ separation from the next highest mode detected. The close match in amplitude measured by each of the three rings indicates that there are limited disruptions to the azimuthal modes, such as standing waves or radial modes. The far field directivity identified by each method shows a good agreement, with peak directivity at 30 degrees for Actran and 34 degrees for both the experiment and COMSOL. All three methods show the main lobe roll-off upstream at $-10 \mathrm{~dB}$ around 12-14 degrees from the geometry axis. The downstream local minimum after the main lobe is found at 54 degrees in both COMSOL and Actran, while the experiment local minimum is at 59 degrees. The experiment and COMSOL match with a local minimum of about $-4 \mathrm{~dB}$ while Actran results is $-6 \mathrm{~dB}$.

A final set of results is presented in Figure 11, this time for $m=6$ and $8000 \mathrm{~Hz}$. The axial wavelength is approximately of the size of the auxiliary inlet and is seen to interact strongly with it. The result from Actran is given in subplot a), and shows the sound propagating out the auxiliary inlet at an angle roughly perpendicular to the axis of the duct. The COMSOL pressure isosurface plot in subfigure b) shows very sharp lobes and a strong upstream directivity. The overhead experimental survey in subfigure c) shows the usual on-axis null, a large forward lobe, another null and then the start of another lobe. This is matched quite well in the COMSOL result, subfigure d), where the null pattern in the center shows an additional azimuthal pattern of six nodes. These features are too small to be resolved by the experimental survey, although they certainly may be present. Similar but larger features were measured for other cases not presented in this report. The size of the null between the main and second lobe is captured in both COMSOL and the experiment at very similar angles, and happens to be right at the extent of the experimental survey. The in-duct mode measurement given in subfigure e) shows the $m=-6$ mode is around $12 \mathrm{~dB}$ stronger than any other modes. This was not the case for all modes measured, and a number of interesting results were identified from the mode measurement ring, but a comprehensive discussion of these results is beyond the scope of the present paper. The radiated sound directivity given in $f$ ) is reasonably consistent between the experiment and COMSOL, with the peak directivity angle of the main lobe found at 37 degrees in Actran, 42 degrees in the experiment and 43 degrees in COMSOL. The null at around 50 degrees is found to agree quite well between the experiment and COMSOL, with Actran being the outlier again. As the density of features in the tone directivity increases, it is reasonable to expect individual features to be more sensitive to differences in the geometry and configuration. 


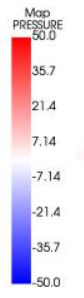

a) Actran pressure field

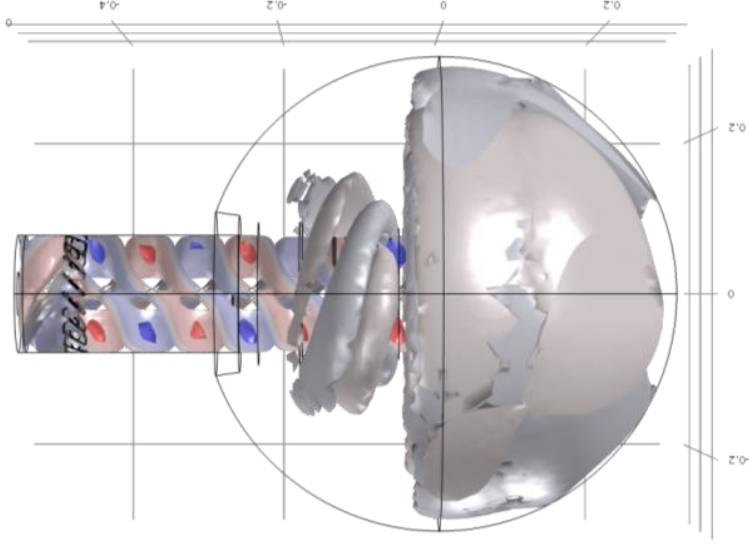

b) COMSOL pressure field

Tone Level, $\mathrm{dB}$

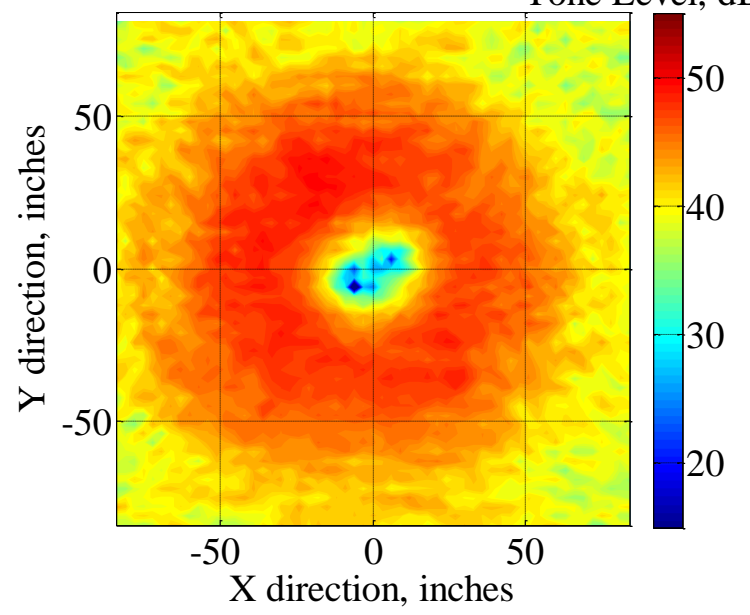

c) Experiment overhead survey

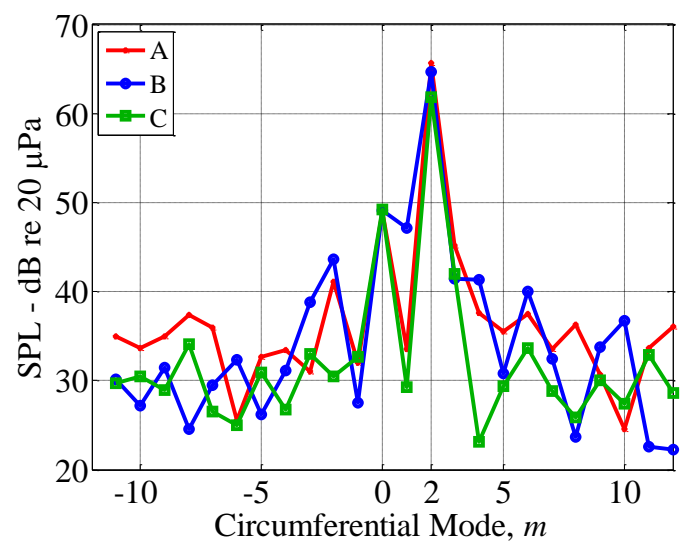

e) In-duct mode array measurement

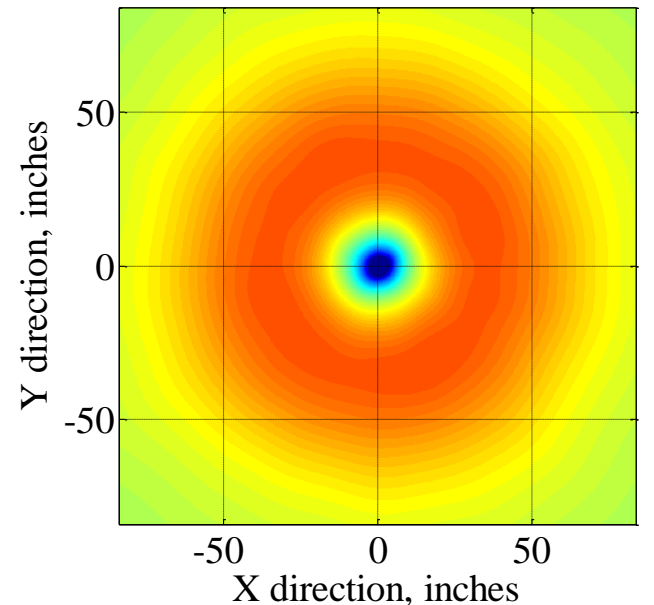

d) COMSOL overhead survey

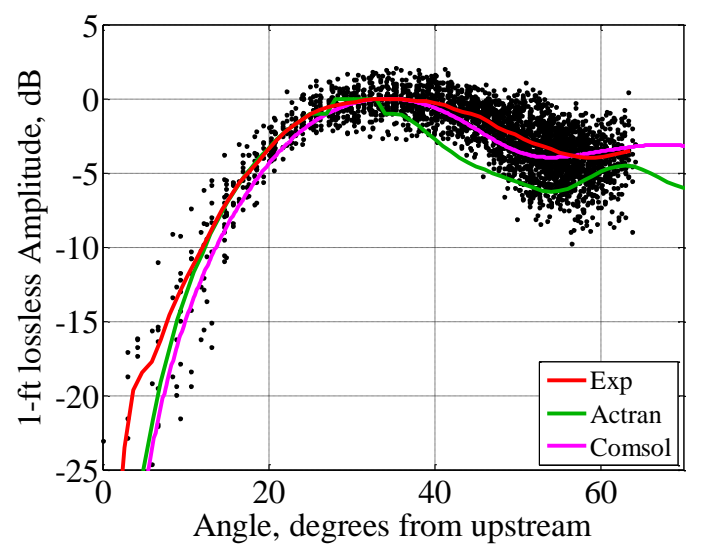

f) Radiated sound directivity

Figure 10-Combined diagnostic results for $m=2,3500 \mathrm{~Hz}$. 

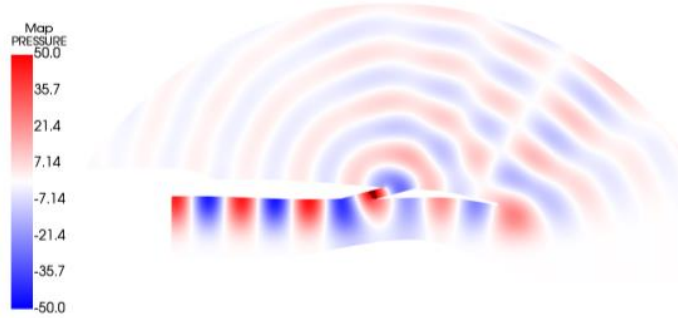

a) Actran pressure field

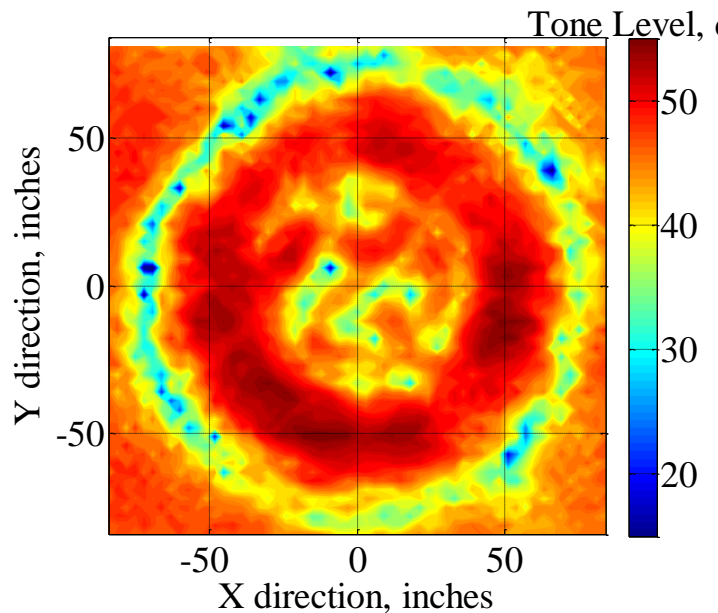

c) Experiment overhead survey

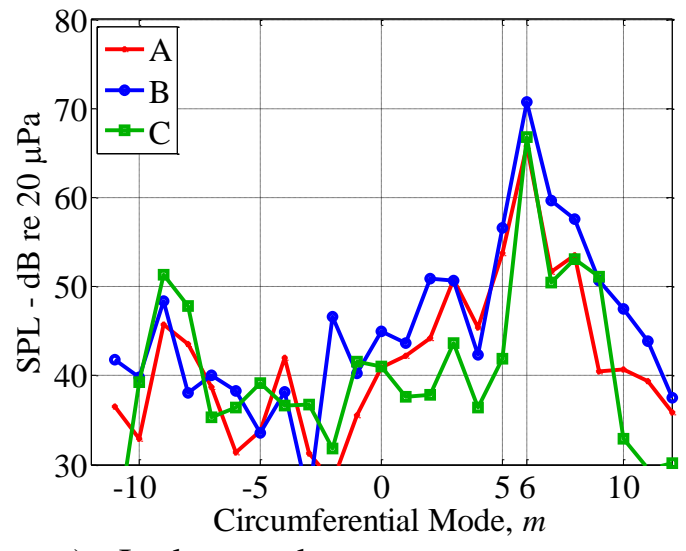

e) In-duct mode array measurement

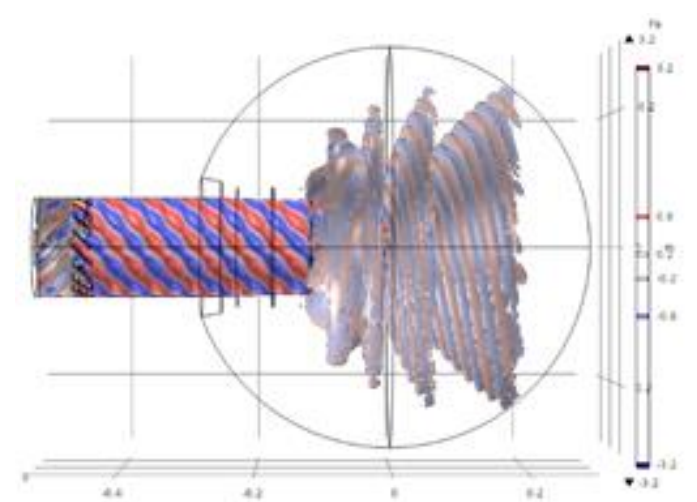

b) COMSOL pressure field

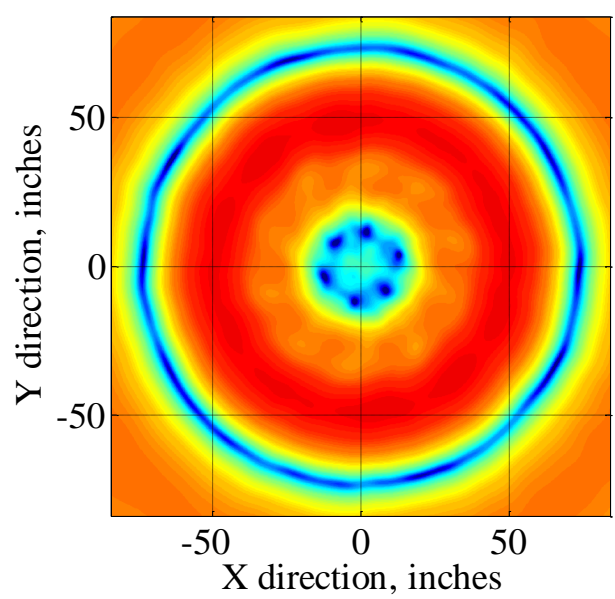

d) COMSOL overhead survey

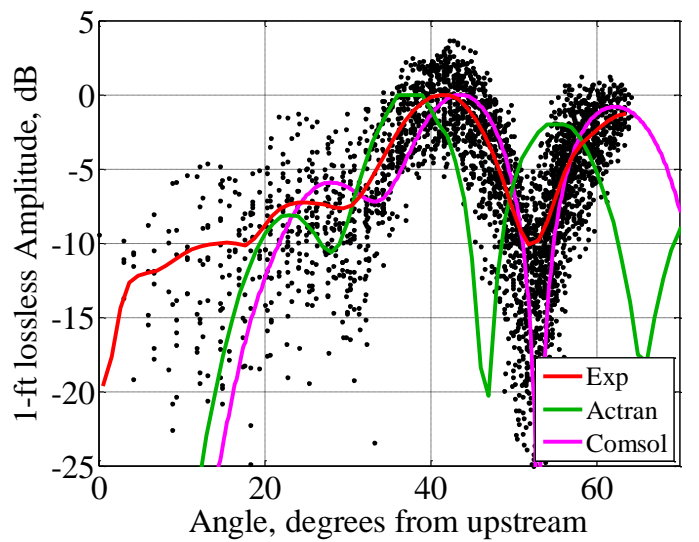

f) Radiated sound directivity

Figure 11 - Combined diagnostic results for $m=6,8000 \mathrm{~Hz}$.

Instead of reporting on all measurements for each mode and frequency combination, a simplified result is given. The angle of the peak noise radiation directivity is given in Figure 12, with the range set to match that of the experiment, 63 degrees from upstream. For all three methods and both modes investigated, the overall trends match well. For $m=2$, the peak radiation angle moves about $20^{\circ}$ aft from 2500 to $5000 \mathrm{~Hz}$. The agreement at $m=2$ is worst at $2500 \mathrm{~Hz}$, but this was already examined in Figure 9 and associated text. The higher frequency tone travels down the duct at a steeper propagation angle, so the main radiation 
lobe is further from the axis. For $m=2$, COMSOL and the experiment match very closely for frequencies of $3000 \mathrm{~Hz}$ and higher. The Actran simulation consistently shows a peak radiation angle around 5 degrees closer to the duct axis. The reason for this has not been identified, but it assumed to be related to one of the details of the experiment that COMSOL incorporates, but that Actran does not. A largely similar story is shown in subfigure $\mathrm{b}$ ), for $m=6$. This time, the COMSOL result does not match the experiment quite as well, consistently having a peak directivity a few degrees closer to the duct axis. The Actran result again shows the most-upstream peaks of the three methods. The experimental data at $9500 \mathrm{~Hz}$ was not acquired correctly and no data at this condition is currently available.

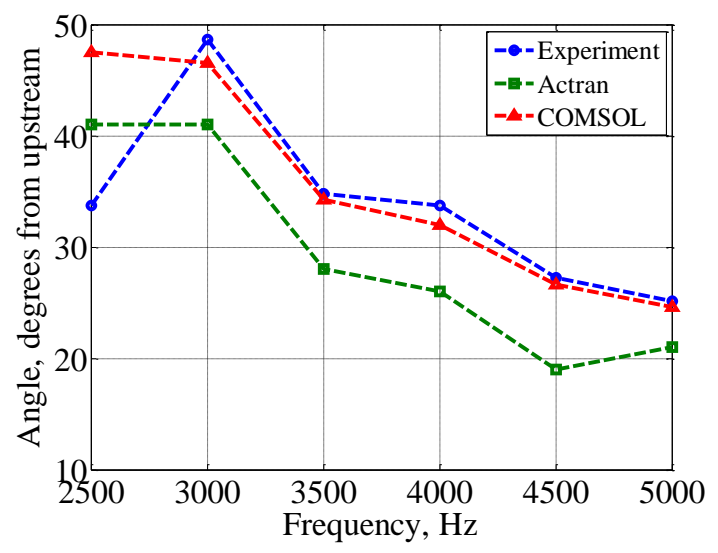

a) $m=2$, peak noise directivity angle

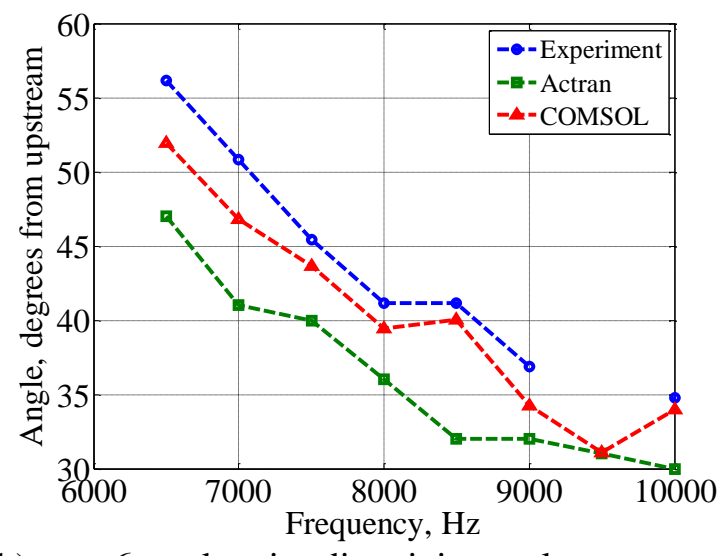

b) $m=6$, peak noise directivity angle

Figure 12 - Peak noise radiation directivity angle found within upstream $63^{\circ}$ for each method.

\section{Discussion}

Despite the authors' intentions to make a simple experimental validation of the acoustic codes, the comparison of various simulations and experiments was consistently complicated. Simply measuring an axisymmetric sound field from a notionally axisymmetric model was found to require careful selection of source parameters. Non-axisymmetric measurements, such as Figure $9 \mathrm{c}$ ) were the norm, rather than the exception. Upon reviewing a considerably larger volume of simulations and experiments than is presented here, the authors came to recognize several reasons a sound field might not be axisymmetric:

- There will doubtless be imperfections in the geometric alignment of the individual pieces of the experiment and the overhead microphone rake. These could cause disruption to the expected spiral sound pattern and manifest in non-axisymmetric sound fields. This effect has not been carefully investigated.

- Multiple azimuthal modes may be excited simultaneously, besides the one being targeted.

- Multiple azimuthal modes can interfere with each other to create standing waves, such as seen in the inner node pattern seen in Figure $11 \mathrm{~d}$ ). Some of these patterns were measured quite convincingly in the experiment and predicted using COMSOL, but these were not included in the present report for brevity. Actran was run in 2-D axisymmetric mode, so periodicity is enforced. Any limitations in the experiment reducing the axisymmetry of the configuration would not be captured by Actran. Individual modes are being inserted in Actran, while the experiment has imperfections that may lead to multiple modes existing when only one is expected.

- Multiple radial modes may be cut on, either at the point of excitation or as the sound propagates down the duct with changing annular cross-section. The inability to control specific radial modes is a limitation in the experiment, where a single ring of actuators does not have control over the radial distribution of sound in the duct. Each radial mode has a different propagation angle and 
therefore radiation lobe. If multiple modes are propagating at once, interference patterns can again result.

- Limitations of the experiment include the use of 18 drivers azimuthally distributed at a single radial location. This limits the mode generation capability to $m=-8$ to $m=8$, although better results might be obtained at lower mode orders. Specific radial modes could not be generated except by restricting the frequency considered to ensure only one mode would be cut on. The drivers were calibrated for amplitude response, but no correction for phase was deemed necessary. This assumption could be revisited.

- The mode measurement device is three rings of 24 microphones. This limits mode measurement to azimuthal modes below $+/-12$ and radial modes to below 2 . A detailed examination of the mode measurement survey was not conducted in the present study

- The overhead survey was conducted at a grid spacing of $76 \mathrm{~mm}$ (3 inches), which limits the resolution of the sound field feature size that can be measured. This would be easily resolved at the expense of additional measurement time.

- The presence of the auxiliary inlet allows the tone noise to exit the model in two locations. Thus a single azimuthal mode can interfere with itself and produce an interference pattern.

For all of the cases presented here, the tone wavelength is roughly equal or larger when compared with the size of the auxiliary inlet. It is apparent that some of the sound produced by the fan is radiated out the auxiliary door, and the peak directivity of this sound can be dramatically different than the sound at the same frequency and mode that propagates out of the main inlet. For an under-wing mounted engine, this sound propagation would be directly towards the ground. The noise from the auxiliary inlet also interferes perfectly with the noise from the main inlet, creating nodes and antinodes which would be experienced as oscillating noise levels by an observer.

\section{Conclusions}

The use of an experiment alongside two computational methods to investigate noise propagating through an axisymmetric spike inlet for a supersonic engine was investigated. In order to compare all three methods, a specific set of test cases was considered. Actran was operated in a 2-D periodic mode, so non-axisymmetric effects could not be captured. COMSOL was limited in calculation size, but was able to incorporate many features of the experiment. The experiment was built using 3-D printed parts, so ideal acoustic qualities and geometric assembly were not guaranteed. For the 14 mode/frequency combinations tested, agreement was typically acceptable and deviations were usually able to be explained. Future work is expected to include a more advanced in-duct mode measurement ring for the experiment. Simulations will be adapted to include flow effects, although this will likely be done in the simulations only. Future work will also consider the potential of acoustic liners to address the noise radiation out of the main and auxiliary inlets and corresponding effect on reducing flyover noise.

\section{Acknowledgments}

This research was supported by the NASA Commercial Supersonics Technology project of the Advanced Air Vehicle Program and the NASA CT Space Grant Consortium. Thanks to NASA GRC Acoustics Branch researcher Dr. Daniel L. Sutliff and Dr. Bruce E. Walker from Channel Island Acoustics for their support designing the experiment and using the UCFANS simulator equipment. 


\section{References}

[1] J. Morgenstern, M. Bounamo, J. Yao, M. Murugappan, U. Paliath, L. Cheung, I. Malcevic, K. Ramakrishman, N. Pastouchenko, T. Wood, S. Martens, P. Viars, T. Tersmette, J. Lee and G. Carrier, "NASA/CR-2015-218719," Advanced Concept Studies for Supersonic Commercial Transports Entering Service in the 2018-2020 Period Phase 2, pp. 1-30, 2015.

[2] C. M. Heath, J. A. Seidel and S. K. Rallabhandi, "Viscous Aerodynamic Shape Optimization with Installed Propulsion Effects," in AVIATION Forum, Denver, Colorado, USA, 2017.

[3] D. L. Sutliff and B. E. Walker, "Characteristics using an Ultrasonic Configurable Fan Artificial Noise Source to Generate Modes - Experimental Measurements and Analytical Predictions," in 20th AIAA/CEAS Aeroacoustics Conference, Atlanta, GA, USA, 2014.

[4] D. L. Sutliff, C. A. Brown and B. E. Walker, "Hybrid Wing Body Shielding Studies Using an Ultrasonic Configurable Fan Artificial Noise Source Generating Simple Modes," in NASA/TM2012-217685, 2012.

[5] D. L. Sutliff, C. A. Brown and B. E. Walter, "Hybrid wing body shielding studies using an ultrasonic configurable fan artificial noise (AIAA 2014-0256)," in 52nd Aerospace Sciences Meeting, National Harbor, MD, USA, 2014.

[6] D. L. Sutliff and B. E. Walker, "Artificial noise systems for parametric studies of turbo-machinery aero-acoustics," International Journal of Aeroacoustics, vol. 15, no. 1-2, pp. 103-130, 2016.

[7] B. A. Cooper, "Design and construction of a convertible hemi/anechoic acoustical laboratory for testing space flight hardware at the NASA Glenn Research Center," The Journal of the Acoustical Society of America, vol. 108, no. 5, pp. 2473-2473, 2000.

[8] D. M. Podboy, J. H. Mirecki, B. E. Walker and D. L. Sutliff, "Recent improvements to the acoustical testing laboratory at the NASA Glenn Research Center (NASA/TM-2014-218110)," NASA, 2014.

[9] COMSOL, AB, "COMSOL Multiphysics, Software Package, Ver. 5.3," Stockholm, Sweeden.

[10] J. W. Slater, "SUPIN: A Computational Tool for Supersonic Inlet Design," in SciTech Forum, San Diego, California, USA, 2016.

[11] F. G. R.P. Woodward and J. Lucas, "AIAA-83-1415 Low Flight Speed Acoustic Results for a Supersonic lnlet with Auxiliary lnlet Doors," A.I.A.A., Cleveland, 1983.

[12] W. Eversman, "Theoretical Models for Duct Acoustic Propagation and Radiation," NASA Report N92-14782, pp. 101-165, 1991. 